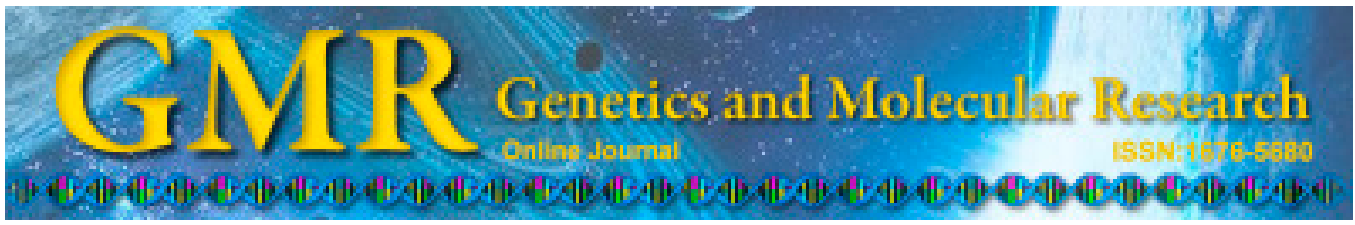

\title{
Role of inflammatory responses in the pathogenesis of human cerebral aneurysm
}

\author{
X.J. Fan ${ }^{1}$, H.D. Zhao', G. Yu' ${ }^{1}$, X.L. Zhong ${ }^{1}$, H. Yao ${ }^{1}$ and Q.D. Yang ${ }^{2}$ \\ ${ }^{1}$ Department of Neurology, \\ The Third Xiangya Hospital of Central South University, \\ Changsha, Hunan, China \\ ${ }^{2}$ Department of Neurology, \\ The Xiangya Hospital of Central South University, \\ Changsha, Hunan, China \\ Corresponding author: Q.D. Yang \\ E-mail: qidongyang2012@126.com
}

Genet. Mol. Res. 14 (3): $9062-9070$ (2015)

Received October 21, 2014

Accepted April 24, 2015

Published August 7, 2015

DOI http://dx.doi.org/10.4238/2015.August.7.15

\begin{abstract}
We aimed to detect expressional profiles of intracellular cell adhesion molecule-1 (ICAM-1), nuclear factor-kappa B (NF- $\mathrm{B}$ ), and monocyte chemoattractant protein-1 (MCP-1) in human cerebral aneurysm, in order to investigate the effect of chronic inflammation on the pathogenesis of cerebral aneurysm. Samples from 40 cases of human cerebral aneurysms diagnosed at our hospital were selected along with 20 normal cerebral artery samples. Western blotting and immunohistochemical (IHC) staining were used to reveal expressional profiles of ICAM-1 and NF- $\mathrm{KB}$ in the aneurysmal wall of patients and normal cerebral artery tissues. Reverse transcription (RT)-PCR was employed to detect changes in transcript levels of MCP-1 mRNA. Western blotting showed significantly higher expressions of ICAM-1 and $\mathrm{NF}-\mathrm{kB}$ in patients with cerebral aneurysm compared to the normal group $(\mathrm{P}<0.01)$, which was consistent with $\mathrm{IHC}$ staining results. RT-PCR revealed significantly higher MCP-1 transcripts in cerebral
\end{abstract}


aneurysm tissues compared to the normal group $(\mathrm{P}<0.01)$, in addition to a positive relationship between ICAM-1 and NF- $\mathrm{KB}$ expression levels. In conclusion, expression levels of ICAM-1, NF- $\mathrm{KB}$, and MCP-1 in patients are significantly elevated, suggesting an enhanced chronic inflammatory response and a significant correlation between inflammatory factors/adhesion molecules and the pathogenesis of cerebral aneurysm.

Key words: Cerebral aneurysm; Inflammatory response; ICAM-1; $\mathrm{NF}-\kappa \mathrm{B} ; \mathrm{MCP}-1$

\section{INTRODUCTION}

As a result of malignant tumors that originate in the bifurcations of brain arteries, cerebral aneurysm has a relatively high mortality and reoccurrence rate. Cerebral aneurysm is the third most common among all cerebrovascular diseases next to cerebral thrombosis and hypertensive cerebral hemorrhage, and is the leading cause for subarachnoid hemorrhage, which severely affects patient health. Cerebral aneurysm can be classified into internal carotid artery aneurysm, anterior cerebral artery aneurysm, middle cerebral artery aneurysm, basilar artery aneurysm, and giant aneurysm based on the localization of the tumor (Schwenk et al., 2010; Michel et al., 2011). Studies have shown that $40 \%$ of all aneurysms occur in the bifurcation of the internal carotid artery, with the highest frequency at the posterior communicating artery bifurcation. Aneurysms occurring in the anterior cerebral artery, including the anterior communicating artery bifurcation, make up $30 \%$ of the cases, while middle artery aneurysm represents $20 \%$ of all cases and mainly occurs at trigeminal sites. Multiple aneurysms, on the other hand, commonly occur in the middle cerebral artery, internal carotid posterior communicating artery, and bifurcation of the ophthalmic artery (Golledge et al., 2010; Martinez-Pinna et al., 2010). Recent studies have improved the understanding about cerebral aneurysm; however, its pathogenesis requires further investigations.

Studies have reported some relationships between inflammation and the occurrence/ progression of cerebral aneurysm, possibly due to the activity of proteinases produced by macrophages and lymphocytes during inflammatory response. For example, matrix metalloproteinases can degrade elastic and collagen fibers in vessel walls, facilitating the occurrence and progression of aneurysm, and finally leading to vessel rupture (van Kuijk et al., 2010; Ramos-Mozo et al., 2012). Monocytes progressively differentiate into macrophages during their migration in blood. Monocyte chemoattractant protein-1 (MCP-1) plays an important role in the differentiation, migration, and induction of inflammatory response of macrophages (Aoki et al., 2010; Wang et al., 2014). For example, MCP-1 can be produced by monocytes, macrophages, fibroblasts, endothelial cells, and B cells stimulated by external factors including LPA, Poly I-C, IL-1, EGF, and some viruses (Liu et al., 2014). Intracellular adhesion molecule-1 (ICAM-1) is a glycoprotein in the immunoglobulin superfamily, and is expressed on the surface of endothelial and epithelial cells at low levels under physiological conditions. Inflammation elevates the expression of ICAM-1, which activates the rolling and stable adhesion of leucocytes to blood vessel walls, as well as leucocytes' stable adhesion and penetration across endothelial cells, migration, and recruitment in the inflammatory site, thereby facili- 
tating inflammatory response (Hochberg, 2010). As one of the most important intracellular nuclear factors involving in the inflammation, NF- $\mathrm{KB}$ modulates the expression of various genes during the early stages of the immune response and during all stages of inflammatory response, thereby participating in immunoreaction, inflammatory response, cell apoptosis and tumor pathogenesis (O'Hare et al., 2010). Studies have revealed critical modulatory functions of ICAM-1, NF- $\mathrm{kB}$, and MCP-1 in inflammatory response induced by multiple distinct factors (Chan et al., 2013).

To further investigate the function of the macrophage and related inflammatory factors ICAM-1, NF- $\mathrm{kB}$, and MCP-1 in human cerebral aneurysm, we selected 40 cases of human cerebral aneurysm diagnosed between January 2010 and January 2014, along with 20 samples of normal cerebral arteries, and measured the expressional profiles of ICAM-1, NF$\kappa \mathrm{B}$ and MCP-1 mRNA. We attempted to provide experimental evidence and a theoretical basis for illustrating the pathogenesis of cerebral aneurysms.

\section{MATERIAL AND METHODS}

\section{Patient information}

Forty cases of human cerebral aneurysm diagnosed at our hospital from January 2010 to January 2014 were selected along with 20 samples of normal cerebral arteries. Locations of cerebral aneurysm included the left posterior communicating artery $(\mathrm{N}=15)$, right posterior communicating artery $(\mathrm{N}=10)$, and anterior communicating artery $(\mathrm{N}=15)$. There were 20 male and 20 female patients, with an average age of 53.8 years. The control group included 10 male and 10 female patients with an average age of 52.8 years. There was no statistical difference in age or gender between the two groups. Samples were collected and frozen for further use. Tissue sections were prepared by the Department of Pathology in our hospital. Written consent was obtained from all patients and their families. The protocol of this study was preapproved by the Ethical Committee at our hospital.

\section{Reagents}

ICAM-1, NF- $\kappa B$, and $\beta$-actin antibodies were purchased from Santa Cruz Biotech (USA). Corp. DAB kit was produced by Thermo Corp (USA). RNA extraction kit and RTPCR kit were products of Promega Corp (USA).

\section{Immunohistochemical (IHC) assay for ICAM-1 and NF-кB}

Tissue slices were de-waxed and rehydrated using xylene and graded ethanol. Endogenous peroxidase activity was quenched by incubation in $3 \% \mathrm{H}_{2} \mathrm{O}_{2}$ at room temperature. After rinsing in distilled water 3-5 times, tissue slices were immersed in $0.01 \mathrm{M}$ citrate buffer $(\mathrm{pH}$ 6.0) and were heated in a microwave oven, followed by cooling and PBS washing 2-3 times for antigen retrieval. Following blocking in 5\% BSA for $30 \mathrm{~min}$ at room temperature, sections were incubated in diluted primary antibody $(1: 500)$ at $4^{\circ} \mathrm{C}$ overnight. After PBS rinsing 3-5 times, secondary antibody in dilution buffer $(1: 300)$ was added for $1-\mathrm{h}$ incubation at $37^{\circ} \mathrm{C}$. Tissue slices were then rinsed 3-5 times, developed using DAB chromogenic substrates, counter- 
stained with hematoxylin, dehydrated in gradient ethanol and xylene, and observed under light microscope. A negative control was also performed in parallel using PBS buffer instead of primary antibody. Yellow or brown granules indicated positive signals of IHC staining. Expression levels of all factors were deducted from average optical density values calculated by the ImagePro Plus image analysis software.

\section{Assay of MCP-1 mRNA level by RT-PCR}

Extraction of RNA from tissue samples, as well as the synthesis of cDNA using 2 $\mu \mathrm{g}$ RNA as the template, was performed by following the manufacturer instructions. Expression level of MCP-1 was assayed using cDNA as the template and $\beta$-actin as an internal reference. Primers for RT-PCR were synthesized by BGI China and are listed in Table 1. Relative level of mRNA was presented as the ratio of optical density values (= MCP - 1/ $\beta$-actin).

\section{Table 1. Primer sequences for RT-PCR}

\begin{tabular}{llc}
\hline Name & Sequence (5'-3') & Length (bp) \\
\hline MCP-1-F & GCTCG CTCAG CCAGA TGCAA T & 257 \\
MCP-1-R & TGGGT TGTGG AGTGA GTGTT C & 473 \\
$\beta$-actin-F & AAATC GTGCG TGACA TTAA & 4 \\
$\beta$-actin- $-\mathrm{R}$ & CTCGT CATAC TCCTG CTTG & \\
\hline
\end{tabular}

\section{Western blotting}

Samples of cerebral aneurysm and normal cerebral blood vessel tissues were lysed, electrophoresed on an SDS-PAGE gel, transferred to membranes, blocked, incubated with primary antibody at $4^{\circ} \mathrm{C}$ overnight, washed 3 times, then incubated in secondary antibody for 1 h. After washing, chromogenic substrate was added to develop the membrane in a dark room. The optical density value of protein bands was analyzed using the Quantity One gel imaging system.

\section{Statistical analysis}

The SPSS 18.0 software package was used to process all collected data, which are reported as means \pm standard deviation. Between-group-comparisons were performed by the Student $t$-test (for two samples) or analysis of variance (ANOVA; for multiple groups). A statistical significance was defined when $\mathrm{P}<0.05$.

\section{RESULTS}

\section{CT angiogram (CTA) of patients}

All patients received CTA examinations to identify the location and volume of aneurysms. Some representative images are shown in Figure 1. 

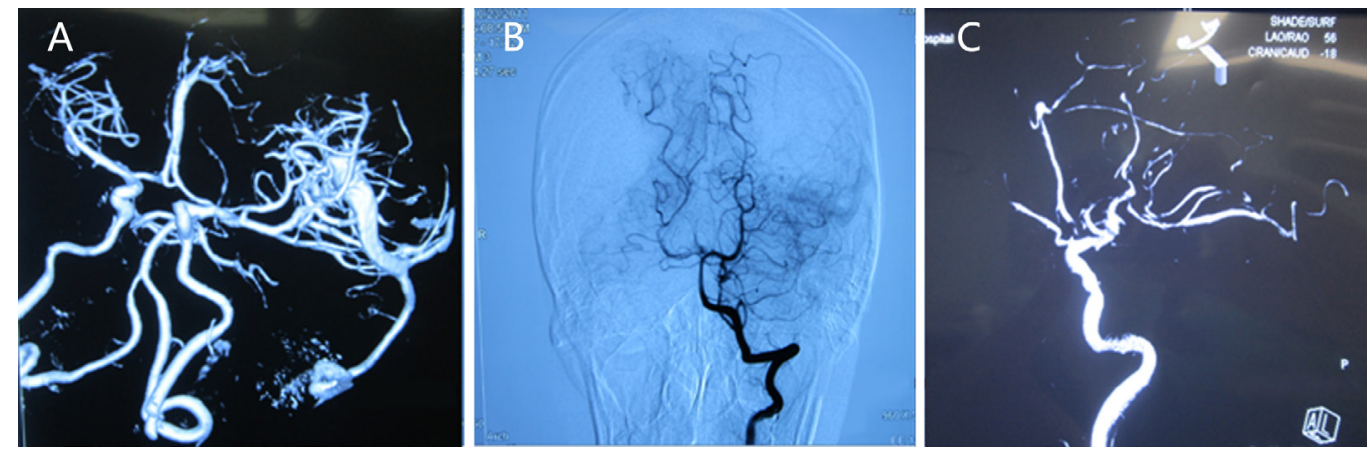

Figure 1. Images of artery aneurysms. A. Anterior communicating artery aneurysm. The body, neck, parent artery, and peripheral blood vessels of the aneurysm can be easily identified. B. Right posterior communicating artery aneurysm, with a clear illustration of the anatomical relationships among anterior, middle, and posterior cerebral arteries and peripheral blood vessels, showing further details of cerebral blood vessels. C. An image of right posterior communicating artery aneurysm, which can be used to observe the tumor neck and peripheral blood vessels from a different view.

\section{Elevated expression of ICAM-1 and NF-кB in aneurysm tissues}

The western blotting method was utilized to analyze the expression of ICAM-1 and $\mathrm{NF}-\kappa \mathrm{B}$ in cerebral aneurysm tissues. Results showed significantly higher ICAM-1 and NF- $\kappa \mathrm{B}$ levels in cerebral aneurysm tissues compared to the normal group $(\mathrm{P}<0.01)$ (Figure 2). This was consistent with IHC staining results showing protein expression profiles of ICAM-1 and $\mathrm{NF}-\kappa \mathrm{B}$, which appeared as dispersive distributions of brown granules (Figure 3): ICAM-1 was expressed in cerebral aneurysm tissues, with brown staining in the cytoplasm or membrane; $\mathrm{NF}-\kappa \mathrm{B}$ also showed positive expression in aneurysm tissues, distributed mainly in the nucleus. As shown in Table 2, optical density values of ICAM-1 and NF- $\kappa$ B in cerebral aneurysm tissues were significantly higher than those in normal samples $(\mathrm{P}<0.01)$.
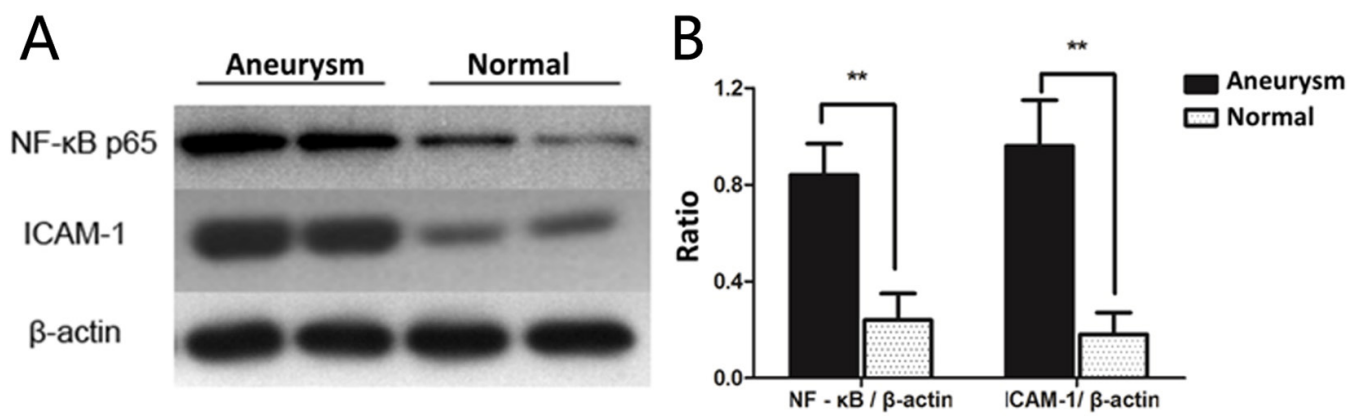

Figure 2. Expression of ICAM-1 and NF- $\mathrm{B}$ in cerebral aneurysms and normal tissues by western blotting. A. Western blotting results showing detection of NF- $\mathrm{kB}$ p 65 and ICAM-1 expression in cerebral aneurysm and normal tissues. The housekeeping gene $\beta$-actin was used as the internal reference. B. Ratios of NF-kB and ICAM-1 levels against $\beta$-actin. NF- $\mathrm{KB}$ and ICAM-1 had significantly elevated expression in cerebral aneurysm compared to normal tissues. ${ }^{* *} \mathrm{P}<0.01$. 


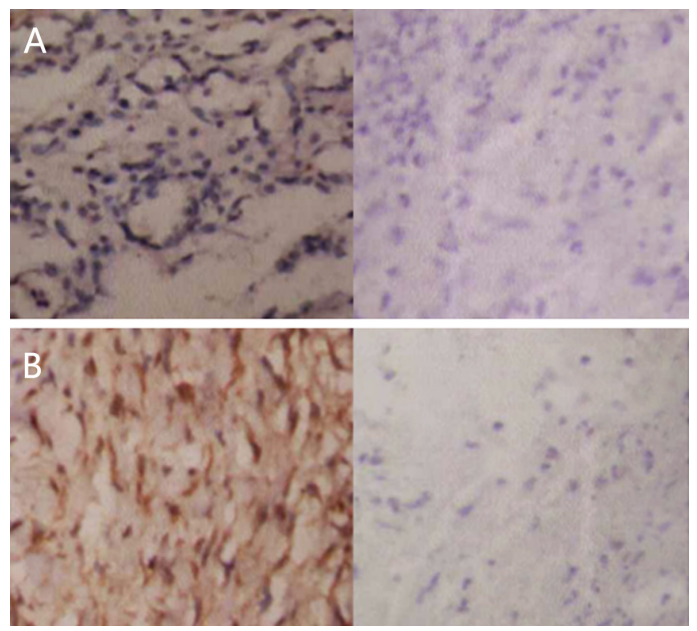

Figure 3. Expression of ICAM-1 and NF- $\mathrm{KB}$ in cerebral aneurysm and normal tissues by IHC staining. A. IHC images (400X) of ICAM-1 in cerebral aneurysm (left) and normal tissues (right). Positive signals of ICAM-1 are shown as yellow or brown colors in the cytoplasm and membrane. B. IHC images (400X) of NF- $\mathrm{KB}$ in cerebral aneurysm (left) and normal tissues (right). Positive signals of NF- $\mathrm{BB}$ are localized in the nucleus and appear brown.

Table 2. Optical density values for expression of ICAM-1 and NF-кB.

\begin{tabular}{|c|c|c|c|}
\hline Group & $\mathrm{N}$ & ICAM-1OD values & $\mathrm{NF}-\kappa \mathrm{B}$ OD values \\
\hline Aneurysm & 40 & $2667.52 \pm 360.38 * *$ & $3867.52 \pm 1260.36^{* *}$ \\
\hline Normal & 20 & $1098.98 \pm 284.32$ & $698.98 \pm 264.32$ \\
\hline$t$ value & & 4.216 & 3.967 \\
\hline$P$ value & & 0.0064 & 0.005 \\
\hline
\end{tabular}

$* * \mathrm{P}<0.01$ compared to the normal group.

\section{RT-PCR assay of MCP-1 mRNA transcripts}

To further compare differences in expression levels of MCP-1 between cerebral aneurysm and normal tissues, the RT-PCR technique was utilized to quantify levels of MCP-1 mRNA using the housekeeping gene $\beta$-actin as the internal reference due to its stable transcription across tissues. We found significantly higher expression of MCP-1 in cerebral aneurysm tissues compared to the normal group, as shown in Figure 4. Further comparison using the optical density value as the index to compare transcript levels of the MCP-1 gene between patients with cerebral aneurysm and the normal group (Figure 5) showed elevated transcript levels of MCP-1 in pathogenic cerebral aneurysm tissues.

\section{DISCUSSION}

Cerebral aneurysm is a common human cerebrovascular disease that frequently develops in the posterior communicating and anterior communicating arteries. As the major cause of subarachnoid hemorrhage and cerebral hematoma, it mainly presents as headache, consciousness disorder, vomiting, and hemiparalysis (Zhong et al., 2013; Zaidat et al., 2014). Without timely and effective treatment, aneurysm has a high chance of rupture, leading to its 
high mortality and disability rates (Zairi et al., 2011; Chalouhi et al., 2013). Epidemiological studies have shown that smoking, drinking, diabetes, and hypertension are major contributors to cerebral aneurysm, whilst its exact pathogenesis still remains unclear. Recent studies about aneurysm have made significant progress regarding its pathogenesis, including underdevelopment of the artery muscular layer, atherosclerosis driven by diabetes, the inflammatory response as peripheral edema, and inflammation occurring in patients with cerebral aneurysm under enhanced scanning (Chalouhi et al., 2013).

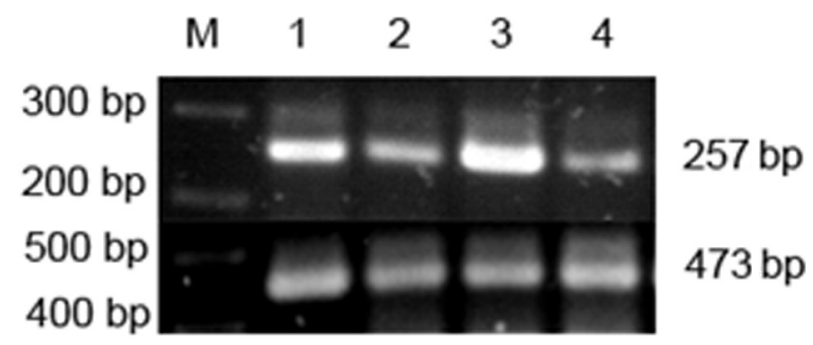

Figure 4. RT-PCR results of MCP-1 mRNA. Lane $M=$ DNA marker; lanes 1 and 3 = cerebral aneurysms; lanes 2 and $4=$ normal tissues.



Figure 5. MCP-1 mRNA levels in cerebral aneurysm and normal tissues. $* \mathrm{P}<0.01$ compared to normal tissues.

One of the significant features of inflammation is gene transcription and expression of inflammatory cytokines, chemoattractant factors, and adhesion molecules. Among immune cells that induce chronic inflammation, the most significant function is exerted by infiltrative macrophages, which can be chemo-attracted by MCP-1 to infiltrate peripheral tissues. Enzymes produced by macrophages can degrade elastic fibers and collagen fibers in the blood vessel wall, leading to the occurrence and even rupture of arterial aneurysm (Wojtkowiak et al., 2011; Wen et al., 2013). The promoter region of the MCP-1 gene includes a binding site for NF- $\mathrm{kB}$, which can modulate the expression of proteins including MCP-1 at the transcriptional level. Furthermore, the gene encoding the adhesion molecule ICAM-1 also has a NF- $\mathrm{kB}$ 
binding site about $200 \mathrm{bp}$ upstream of its translation initiation site. Therefore, NF- $\mathrm{kB}$ regulates the expression of both MCP-1 and ICAM-1. In support of this hypothesis, studies have shown highly positive relationships between ICAM-1 or MCP-1 and NF- $\mathrm{KB}$ expression levels (Papayianni et al., 2002; Tumur et al., 2010; Lu et al., 2012).

To further study the relationship between pathogenesis, progression of cerebral aneurysm, and inflammatory response, this study investigated the inflammatory factors ICAM-1 and NF- $\mathrm{kB}$, which are known to be involved in inflammatory response and cerebral aneurysm, in addition to the expressional profile of the macrophage-specific protein MCP-1 during the progression of cerebral aneurysm. Our results showed significantly higher ICAM-1, NF- $\mathrm{kB}$, and MCP-1 expression levels in cerebral aneurysm tissues compared to normal tissues (P $<0.05)$, suggesting a close relationship between inflammatory response and the occurrence and development of cerebral aneurysm. This study, however, only focused on the changes in inflammatory factor expression in cerebral aneurysm and omitted other aspects of the pathogenesis. Therefore, we propose a role for the inflammatory response in cerebral aneurysm, although its detailed pathogenesis still requires further investigation.

\section{Conflicts of interest}

The authors declare no conflict of interest.

\section{ACKNOWLEDGMENTS}

We thank the anonymous reviewers for reviewing this manuscript.

\section{REFERENCES}

Aoki T, Kataoka H, Nishimura M, Ishibashi R, et al. (2010). Ets-1 promotes the progression of cerebral aneurysm by inducing the expression of MCP-1 in vascular smooth muscle cells. Gene Ther. 17: 1117-1123.

Chalouhi N, Hoh BL, and Hasan D (2013). Review of cerebral aneurysm formation, growth, and rupture. Stroke 44: 3613-3622.

Chan A, Orme RP, Fricker RA and Roach P (2013). Remote and local control of stimuli responsive materials for therapeutic applications. Adv. Drug Deliv. Rev. 65: 497-514.

Golledge J, Clancy P, Moran C, Biros E, et al. (2010). The novel association of the chemokine CCL22 with abdominal aortic aneurysm. Am. J. Pathol. 176: 2098-2106.

Hochberg MC (2010). Seminars in Arthritis and Rheumatism enters a new decade. Semin. Arthritis. Rheum. 39: 223.

Liu YF, Zhang Y, Dai D and Xu Z (2014). Expression of NF-kappaB, MCP-1 and MMP-9 in a Cerebral Aneurysm Rabbit Model. Can. J. Neurol. Sci. 41: 200-205.

Lu Y, Zhu X, Liang GX, Cui RR, et al. (2012). Apelin-APJ induces ICAM-1, VCAM-1 and MCP-1 expression via NFkappaB/JNK signal pathway in human umbilical vein endothelial cells. Amino Acids 43: 2125-2136.

Martinez-Pinna R, Barbas C, Blanco-Colio LM, Tunon J, et al. (2010). Proteomic and metabolomic profiles in atherothrombotic vascular disease. Curr. Atheroscler Rep. 12: 202-208.

Michel JB, Martin-Ventura JL, Egido J, Sakalihasan N, et al. (2011). Novel aspects of the pathogenesis of aneurysms of the abdominal aorta in humans. Cardiovasc. Res. 90: 18-27.

O’Hare AM, Fanning NF, Ti JP, Dunne R, et al. (2010). HydroCoils, occlusion rates, and outcomes: a large single-center study. Am. J. Neuroradiol. 31: 1917-1922.

Papayianni A, Alexopoulos E, Giamalis P, Gionanlis L, et al. (2002). Circulating levels of ICAM-1, VCAM-1, and MCP-1 are increased in haemodialysis patients: association with inflammation, dyslipidaemia, and vascular events. Nephrol. Dial. Transplant. 17: 435-441.

Ramos-Mozo P, Rodriguez C, Pastor-Vargas C, Blanco-Colio LM, et al. (2012). Plasma profiling by a protein array approach identifies IGFBP-1 as a novel biomarker of abdominal aortic aneurysm. Atherosclerosis 221: 544-550. 
Schwenk JM, Igel U, Kato BS, Nicholson G, et al. (2010). Comparative protein profiling of serum and plasma using an antibody suspension bead array approach. Proteomics 10: 532-540.

Tumur Z, Shimizu H, Enomoto A, Miyazaki H, et al. (2010). Indoxyl sulfate upregulates expression of ICAM-1 and MCP1 by oxidative stress-induced NF-kappaB activation. Am. J. Nephrol. 31: 435-441.

van Kuijk JP, Flu WJ, Chonchol M, Bax JJ, et al. (2010). Metabolic syndrome is an independent predictor of cardiovascular events in high-risk patients with occlusive and aneurysmatic peripheral arterial disease. Atherosclerosis 210: 596601.

Wang Q, Ren J, Morgan S, Liu Z, et al. (2014). Monocyte chemoattractant protein-1 (MCP-1) regulates macrophage cytotoxicity in abdominal aortic aneurysm. PLoS One 9: e92053.

Wen H, Guo J, Chang B and Yang W (2013). pH-responsive composite microspheres based on magnetic mesoporous silica nanoparticle for drug delivery. Eur. J. Pharm. Biopharm. 84: 91-98.

Wojtkowiak JW, Verduzco D, Schramm KJ and Gillies RJ (2011). Drug resistance and cellular adaptation to tumor acidic pH microenvironment. Mol. Pharm. 8: 2032-2038.

Zaidat OO, Castonguay AC, Teleb MS, Asif K, et al. (2014). Middle cerebral artery aneurysm endovascular and surgical therapies: comprehensive literature review and local experience. Neurosurg. Clin. N. Am. 25: 455-469.

Zairi F, De Saint Denis T, Thines L, Bourgeois P, et al. (2011). Ruptured cerebral oncotic aneurysm from choriocarcinoma: report of two cases and review of the literature. Acta Neurochir. 153: 353-357.

Zhong Z, Sun Y, Lin D, Sun Q, et al. (2013). Surgical treatment of brain tumor coexisted with intracranial aneurysm - case series and review of the literature. Neurosurg. Rev. 36: 645-656. 